\title{
New Synthetic Route for Dextran Graft Copolymers Containing Thermo- Responsive Polymers
}

\author{
Kang Moo HuH, Yoshikazu Kumashiro, Tooru OoYA, and Nobuhiko YuI ${ }^{\dagger}$ \\ School of Materials Science, Japan Advanced Institute of Science and Technology, \\ 1-1 Asahidai, Tatsunokuchi, Ishikawa 923-1292, Japan
}

(Received July 31, 2000; Accepted October 2, 2000)

\begin{abstract}
KEY WORDS Dextran / Graft Copolymer / Poly( $N$-isopropylacrylamide-co- $N, N$-dimethylacrylamide) / Thermo-Responsive Grafts / Enzymatic Degradation /
\end{abstract}

Dextran and its derivatives have been extensively studied for biomedical applications due to its biocompatibility and biodegradability. ${ }^{1,2}$ Many studies have been carried out on activation and modification procedures to introduce functional groups onto degradable chains and observed modified properties. Enzymatic degradations were carefully investigated for several applications such as blood substitutes and drug carriers. ${ }^{2,3}$ Enzymatic biodegradability was often reduced or prevented with some chemical modifications involving substitution and crosslinking reactions.

We have modulated the enzymatic degradation in polymeric systems to exhibit a multi-stimuli-responsive fashion. Several polymeric systems have been developed where degradation is dual-stimuli-responsive or modulated with temperature. ${ }^{4-7}$ Such polymeric systems would be very useful for drug delivery because they can respond to more than two stimuli simultaneously and selectively, while most stimuli-responsive polymers are designed to be responsive to a single stimulus. We synthesized a dextran grafted with poly $(N$ isopropylacrylamide-co- $N, N$-dimethylacrylamide)s, poly(NIPAAm-co-DMAAm)s, which is important as a building-block for the development of new multi-stimuliresponsive polymeric systems. ${ }^{8}$ However, adequate control of structural variables such as graft number and length is not easy due to low reactivity of related polymeric reactions and the small number of reactive sites in activated dextrans.

In this study, a new synthetic route to introduce thermo-responsive grafts onto dextrans was developed so that changing synthetic conditions can easily control the structures of graft copolymers such as graft length and number. This method involves the synthesis of semitelechelic poly(NIPAAm-co-DMAAm)s with a hydrazide end-group, and coupling reaction with $p$-nitrophenyl chloroformate-activated dextran. Dextran graft copolymers were found to exhibit an anomalous enzymatic degradation with temperature change.

\section{EXPERIMENTAL}

Synthesis of Semitelechelic Poly(N-isopropylacrylamideco-N,N-dimethylacrylamide), $\left(\mathrm{STP}-\mathrm{NHNH}_{2}\right)$
Semitelechelic polymers were obtained by a synthetic route shown in Figure 1. NIPAAm, DMAAm, methyl 3mercaptopropionate and azobisisobutyronitrile (AIBN) were dissolved in methanol with a predetermined feed ratio of monomers and chain transfer agent as listed in Table I. The solution was bubbled with nitrogen gas for $30 \mathrm{~min}$ and kept at $50^{\circ} \mathrm{C}$ with stirring for $24 \mathrm{~h}$. Finally, the solution was concentrated and precipitated with excessive cold diethyl ether. The final product, STP$\mathrm{COOCH}_{3}$, was obtained by filtration and drying in a vacuum oven for $24 \mathrm{~h}$. The hydrazinolysis reaction of STP$\mathrm{COOCH}_{3}$ to STP-NHNH${ }_{2}$ was performed with hydrazine monohydrate. STP- $\mathrm{COOCH}_{3}$ solution in methanol was added dropwise to methanol solution containing excessive hydrazine monohydrate with stirring. After $5 \mathrm{~h}$ reflux, the product was isolated by precipitation into excessive diethyl ether, followed by dialysis against water (MWCO 500) for 3 days and lyophilization.

\section{Preparation of p-Nitrophenyl Chloroformate-Activated Dextran}

The activation reaction of dextran with $p$-nitrophenyl chloroformate was performed in the presence of DMAP (4-dimethylaminopyridine). Dextran (4 g, $0.1 \mathrm{mmol}$ ), $p$ nitrophenyl chloroformate $(4.35 \mathrm{~g}, 21.6 \mathrm{mmol})$ and small amount of DMAP as a catalyst were dissolved in $250 \mathrm{~mL}$ of a mixture of DMSO and pyridine (1/1 in vol. ratio) and kept at $0^{\circ} \mathrm{C}$ for $8 \mathrm{~h}$. The product was precipitated in ethyl alcohol and filtered. The final product was washed two times with ethyl alcohol and dried in vacuo for $24 \mathrm{~h} . p$ Nitrophenyl groups in the activated dextran were confirmed by ${ }^{1} \mathrm{H}$ NMR characterization. The number of $p$ nitrophenyl groups in the activated dextran was 20 per 100 glucose units by comparing integration values from four aromatic protons in the $p$-nitrophenyl groups ( $\delta=$ 7.54 and $8.30 \mathrm{ppm}$ ) and anomeric protons of glucose units in dextran ( $\delta=4.66 \mathrm{ppm})$.

\section{Synthesis of Dextran Graft Copolymers}

Graft copolymers were prepared by coupling reactions between the $p$-nitrophenyl chloroformate-activated dextrans and semitelechelic polymers as shown in Figure 1. For an example, $1 \mathrm{~g}$ of activated dextran $(0.02 \mathrm{mmol})$ and $0.76 \mathrm{~g}$ of STP-NHNH $\mathrm{NH}_{2}(0.2 \mathrm{mmol})$ were dissolved in

${ }^{\dagger}$ To whom correspondence should be addressed (Tel: +81-761-51-1642, Fax: +81-761-51-1645, E-mail: yui@jaist.ac.jp). 


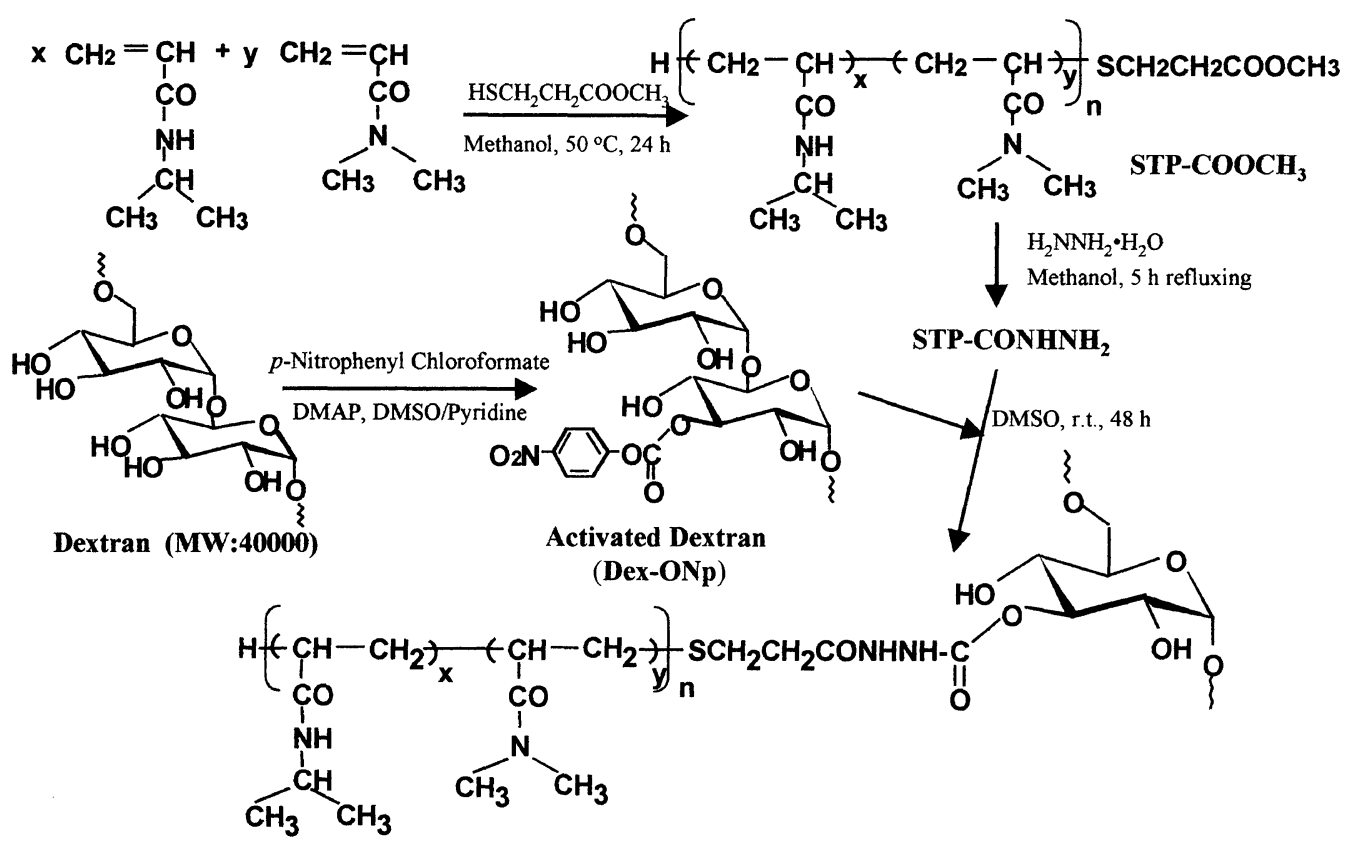

Figure 1. Synthetic scheme for dextran graft copolymers.

$60 \mathrm{~mL}$ of DMSO. After reaction at room temperature for $48 \mathrm{~h}$, the reaction mixture was precipitated in diethyl ether and dried in vacuo. The dried product was dissolved in distilled water and dialyzed $(\mathrm{MWCO}=15000)$ against water to remove unreacted STP- $\mathrm{NHNH}_{2}$ for 3 days and lyophilized. The residual $p$-nitrophenyl groups were hydrolyzed and removed in $0.01 \mathrm{M} \mathrm{NaOH}$ solution during dialysis.

\section{RESULTS AND DISCUSSION}

Semitelechelic poly(NIPAAm-co-DMAAm)s, STPs, were obtained by polymerization of NIPAAm and DMAAm in the presence of methyl 3-mercaptoethanol as chain transfer agent. The results are summarized in Table I. STPs having different molecular weights were obtained by varying feed ratios between the chain transfer agent and monomers. Changing the molar ratio, $[\mathrm{S}] /[\mathrm{M}]$, controlled the MWs of STPs. MW decreased with an increasing amount of chain transfer agent. To compare the effects of the chain transfer agent to STP MWs, STPs were synthesized under the same conditions using mercaptoethanol instead of methyl 3-mercaptoethanol. As shown in Figure 2, methyl 3-mercaptopropionate was more effective to control MWs of STPs than mercaptoethanol. Hydrazinolysis reaction of $\mathrm{STP}-\mathrm{COOCH}_{3}$ to STP- $\mathrm{NHNH}_{2}$ was traced by ${ }^{1} \mathrm{H}$ NMR characterization and the peak from the protons of methyl ester groups ( $\delta$ $=3.53 \mathrm{ppm}$ ) clearly disappeared after hydrazinolysis, indicating complete reaction of methyl ester groups of STP $-\mathrm{COOCH}_{3}$. This reaction is further supported by the fact that MWs determined by titration of the terminal amino-group after hydrazinolysis showed almost the same values with those of $\mathrm{STP}-\mathrm{COOCH}_{3}$, as presented in Table I.

Dextran graft copolymers were synthesized through coupling reaction between activated dextran and STP$\mathrm{NHNH}_{2}$. The results of graft copolymers are summa-

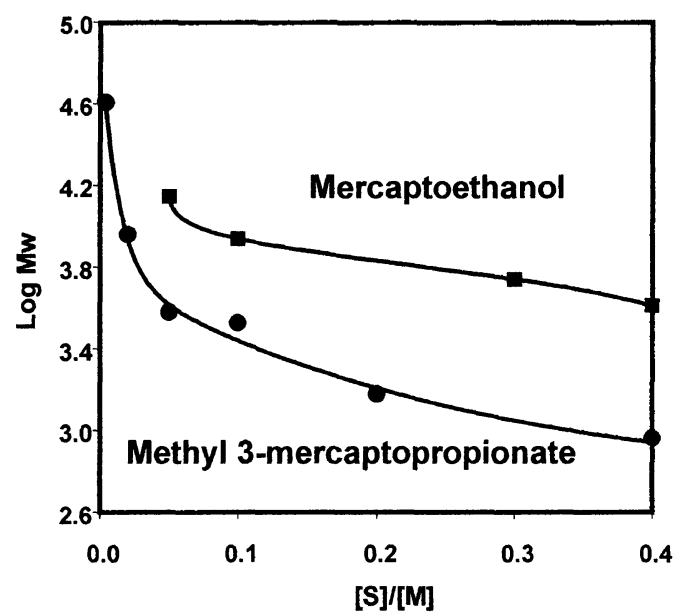

Figure 2. Molecular weight dependence of semitelechelic poly(NIPAAm-co-DMAAm)s on feed ratio of chain transfer agent to monomer.

rized in Table II. Various graft copolymers having different graft lengths and numbers were obtained by simple variation in feed compositions. The number of grafts increased proportionally to feed amount of STP. Chemical compositions and number of grafts of the graft copolymers were determined by ${ }^{1} \mathrm{H}$ NMR characterization. A typical ${ }^{1} \mathrm{H}$ NMR spectrum of the graft copolymers is shown in Figure 3 and characteristic peaks from two components of dextran main-chains and poly(NIPAAmco-DMAAm) grafts were well defined.

In the previous study using coupling between aminoterminated poly(NIPAAm-co-DMAAm)s and carboxymethyl dextran, we could not induce large graft numbers (only $2-4$ grafts per dextran). The present synthetic method, in contrast, using semitelechelic poly(NIPAAm-co-DMAAm)s with a hydrazide end-group which is more reactive than amino end-groups, and $p$ - 
K. M. HuH et al.

Table I. Results for semitelechelic poly(NIPAAm-co-DMAAm)

\begin{tabular}{|c|c|c|c|c|c|c|c|c|}
\hline \multirow{2}{*}{ Samples } & NIPAAm & DMAAm & \multirow{2}{*}[\mathrm{S}]{$/[\mathrm{M}]^{\mathrm{a}}$} & AIBN & \multirow{2}{*}{$M_{\mathrm{n}}^{\mathrm{b}}$} & \multirow{2}{*}{$M_{\mathrm{n}}^{\mathrm{c}}$} & \multirow{2}{*}{$\frac{\text { LCST }^{\mathrm{d}}}{{ }^{\circ} \mathrm{C}}$} & \multirow{2}{*}{$\frac{\text { Yield }}{\%}$} \\
\hline & $\mathrm{g}$ & $\mathrm{g}$ & & $\mathrm{g}$ & & & & \\
\hline STP-1 & 4.1 & 0.9 & 0.4 & 0.148 & 920 & - & 43.2 & 15 \\
\hline STP-2 & 8.2 & 1.8 & 0.2 & 0.296 & 1510 & 1630 & 40.1 & 40 \\
\hline STP-3 & 4.1 & 0.9 & 0.1 & 0.148 & 3370 & 3670 & 40.0 & 50 \\
\hline STP-4 & 8.2 & 1.8 & 0.05 & 0.296 & 3790 & 4070 & 40.5 & 85 \\
\hline STP-5 & 4.1 & 0.9 & 0.02 & 0.148 & 9140 & 10320 & 41.0 & 92 \\
\hline STP-6 & 4.1 & 0.9 & 0.004 & 0.148 & 40570 & - & 40.4 & 90 \\
\hline
\end{tabular}

${ }^{a}$ Molar ratio in feed of chain transfer agent and monomer. ${ }^{b}$ Molecular weight calculated from peak integration of ${ }^{1} \mathrm{H}$ NMR spectra. ${ }^{c}$ Molecular weight determined by titration of the terminal amino group after hydrazinolysis. ${ }^{\mathrm{d}}$ Lower critical solution temperatures of aqueous solutions of STP- $\mathrm{COOCH}_{3}$ (PBS 7.4, $2 \mathrm{wt} \%$ ) measured by UV-VIS light transmittance.

Table II. Synthetic results of dextran graft copolymers

\begin{tabular}{|c|c|c|c|c|c|}
\hline Samples & $\frac{\mathrm{Dex}^{-O N p^{a}}}{\mathrm{mmol}}$ & $\frac{\mathrm{STP}^{\mathrm{b}}}{\mathrm{mmol}}$ & $M_{\mathrm{n}}$ of $\mathrm{Graft}^{\mathrm{c}}$ & $\mathrm{Ng}^{\mathrm{d}}$ & $M_{\mathrm{n}}^{\mathrm{e}}$ \\
\hline GC-1 & 0.02 & 0.2 & 3400 & 7.4 & 64900 \\
\hline GC-2 & 0.02 & 0.2 & 3800 & 8.0 & 70200 \\
\hline GC-3 & 0.02 & 0.1 & 3800 & 4.4 & 56700 \\
\hline GC-4 & 0.02 & 0.6 & 1510 & 17.6 & 66600 \\
\hline GC-5 & 0.02 & 0.2 & 1510 & 8.2 & 52400 \\
\hline GC-6 & 0.02 & 0.1 & 1510 & 4.8 & 47200 \\
\hline
\end{tabular}

${ }^{a} p$-Nitrochloroformate-activated dextran with 20 -nitrophenyl groups per 100 glucose units. ${ }^{\mathrm{b}}$ Molar amount of poly(NIPAAm-coDMAAm)- $\mathrm{NH}_{2}$ in feed. ${ }^{\mathrm{c}}$ Number average molecular weight of graft or poly(NIPAAm-co-DMAAm). ${ }^{\mathrm{d}}$ Average number of grafts calculated from peak integration of ${ }^{1} \mathrm{H}$ NMR spectra. ${ }^{e}$ Number average molecular weight of graft copolymers calculated from the peak integration of ${ }^{1} \mathrm{H}$ NMR spectra.

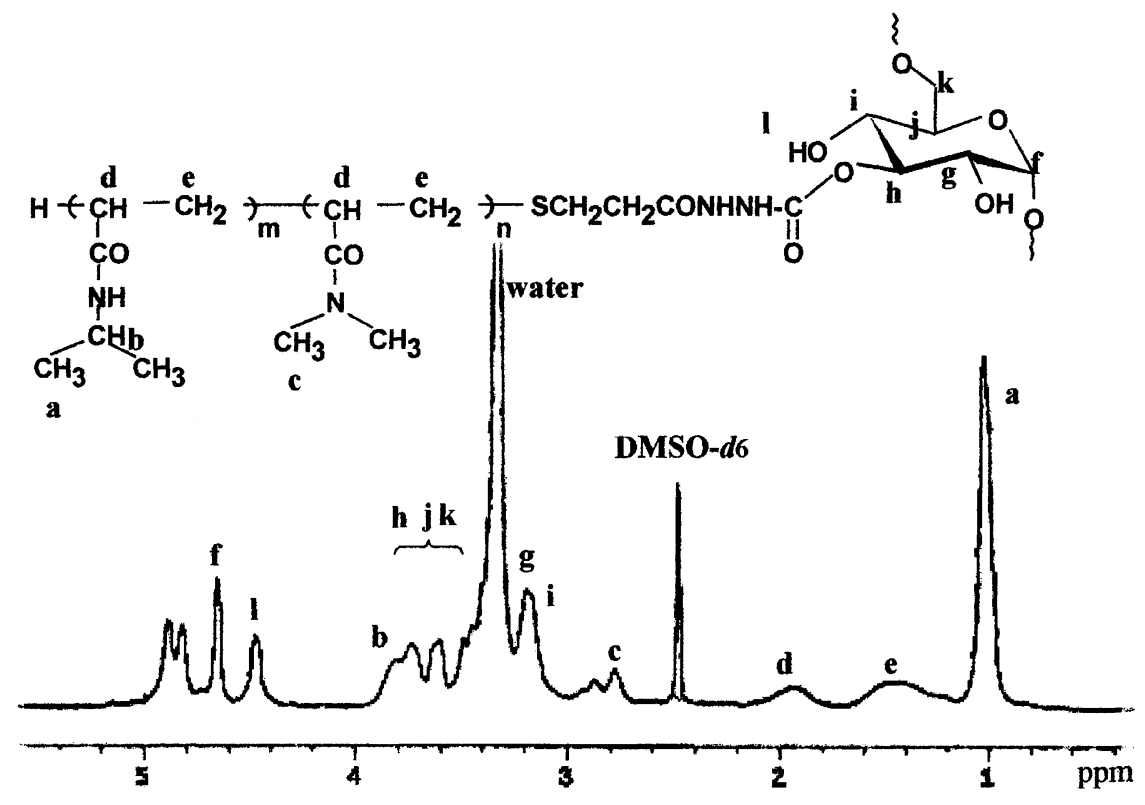

Figure 3. ${ }^{1} \mathrm{H}$ NMR characterization of a dextran graft copolymer.

nitrophenyl chloroformate-activated dextran makes it more convenient to control graft length ( $\mathrm{MW}=920$ $40570 \mathrm{~g} \mathrm{~mol}^{-1}$ ) and number (4-18 grafts per dextran) for modulating the degradation.

Poly(NIPAAm) and its copolymer exhibit clouding at elevated temperature or lower critical solution temperature (LCST) in aqueous solution. LCSTs of STPs were measured by observing change in optical transparency in PBS. The polymers are expected to show higher LCSTs than that of poly(NIPAAm) homopolymer (about $32^{\circ} \mathrm{C}$ ) due to hydrophilic contribution of DMAAm as comonomer. LCST measurements by transmittance of visible light are summarized in Table I. Semitelechelic polymers except for STP-1 exhibit almost the same clouding points around $40^{\circ} \mathrm{C}$ despite different molecular weights. However, STP-1 showed relatively higher LCST, presumably due to its lowest molecular weight. LCST of a poly(NIPAAm) shifts to higher or lower temperature region by the introduction of hydrophilic or hydrophobic comonomers. Thermo-responsiveness of the 


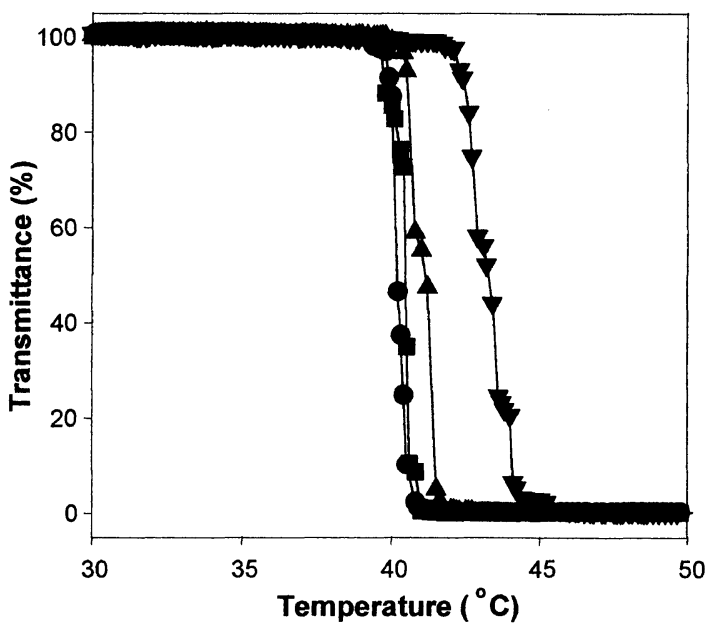

Figure 4. LCSTs of aqueous solutions of semitelechelic polymer and dextran graft copolymers (PBS 7.4, 2 wt\%). $0:$ STP-2, $M_{\mathrm{n}}=$ 1510; $\mathbf{\square}: \mathrm{STP}-\mathrm{NHNH}_{2} ; \mathbf{\Lambda}: \mathrm{GC}-4, \mathrm{Ng}=17.6 ; \boldsymbol{\nabla}: \mathrm{GC}-6, \mathrm{Ng}=4.8$.

STPs is thus adjustable for specific uses.

LCSTs of semitelechelic poly(NIPAAm-co-DMAAm) (STP-2), STP-COOCH 3 , and STP-NHNH ${ }_{2}$, and graft copolymers (GC-4 and GC-5) were compared. As shown in Figure 4, there was no significant difference due to change in end-groups, from STP- $\mathrm{COOCH}_{3}$ to STP$\mathrm{NHNH}_{2}$, in STP-2 but graft copolymers showed increased LCSTs than the corresponding STPs. Graft copolymer with higher graft number showed the lower LCST, due to locally increased thermosensitive grafts along dextran main-chains.

The previous study confirmed no significant change in hydration-dehydration of thermo-responsive poly(NIPAAm-co-DMAAm) after grafting reaction with dextran. ${ }^{8}$ Such grafts should modulate physical and biological properties, especially enzymatic degradation of dextran main-chains in response to temperature change. The degradation of graft copolymers, GC-2, GC-5, and GC-7, having similar graft numbers but different graft lengths, $M_{\mathrm{n}}=3800,1510$, and 9140 , with dextranase was compared by observing change in solution viscosity. Large decrease in solution viscosity from degradation was observed above LCST but not so significant below
LCST. This was most remarkable for GC-7 that has the longest grafts $\left(M_{\mathrm{n}}=9140\right)$. Enzymatic degradation thus proceeds above LCST but is prevented below LCST, due to steric hindrance of enzymatic accessibility by grafted chains. A dependence on the number of grafts was noted and thus enzymatic degradation of the dextran graft copolymer may be modulated by temperature change, which causes hydration-dehydration transition of thermo-responsive grafts. Detailed study of modulated degradation is now in progress. The results will be reported in another paper.

The present synthetic route should be very useful to introduce poly(NIPAAm) or other radical polymers into dextran or even other polysaccharides as grafts, to provide well-defined structure for graft copolymers. Such tailor-made graft copolymers, consisting of biodegradable polysaccharides and stimuli-responsive grafts, may serve as building blocks for designing multi-stimuliresponsive hydrogels.

Acknowledgment. This study was financially supported by a Grant-in-Aid for Scientific Research for Priority Areas "Molecular Synchronization for Construction of New Materials System", from the Ministry of Education, Science, Sports, and Culture, Japan.

\section{REFERENCES}

1. K. Hoste, D. Bruneel, A. D. Marre, F. D. Schrijver, and E. Schacht, Macromol. Rapid Commun., 15, 697 (1994).

2. W. N. E. van Dijk-Wolthuis, O. Franssen, H. Talsma, M. J. van Steenbergen, J. J. Kettenes-van den Bosch, and W. E. Hennink, Macromolecules, 28, 6317 (1995).

3. O. Franssen, R. D. Van Ooijen, D.de Boer, R. A. A. Maes, and W. E. Hennink, Macromolecules, 32, 2896 (1999).

4. M. Kurisawa and N. Yui, Macromol. Chem. Phys., 199, 1547 (1998).

5. M. Kurisawa, M. Terano, and N. Yui, J. Biomater. Sci., Polym. Ed., 8, 691 (1997).

6. M. Kurisawa, Y. Matsuo, and N. Yui, Macromol. Chem. Phys., 199, 705 (1998).

7. M. Kurisawa and N. Yui, Macromol. Chem. Phys., 199, 2613 (1998).

8. K. M. Huh, J. Hashi, T. Ooya, and N. Yui, Macromol. Chem. Phys., 201, 613 (2000). 